\title{
Segment-based Code Assignment in High Speed Downlink Packet Access Networks
}

\author{
Wen-Hsing Kuo and Wanjiun Liao \\ Department of Electrical Engineering \\ National Taiwan University \\ Taipei, Taiwan, China \\ Email: wjliao@cc.ee.ntu.edu.tw
}

\begin{abstract}
In this paper, we study the code assignment problem in High Speed Downlink Packet Access (HSDPA) Networks. In HSDPA, the spreading factor of codes is fixed at 16 , and the maximum number of bits reserved for code assignment transmitted on the control channel is limited to 64 bits. This high control overhead may lead to inefficiencies in HSDPA systems when the number of mobile nodes in a cell becomes large. Existing code assignment mechanisms allow codes with different spreading factors to be allocated, rendering them inadequate for HSDPA systems. In this paper, a new segment-based code assignment mechanism is proposed. In our mechanism, 16 codes are grouped into several segments. Since the number of codes in cach segment is reduced, the corresponding signaling overhead is also reduced. The load of each channel is then balanced in each segment so as to maximizing the system performance.
\end{abstract}

\section{INTRODUCTION}

High-Speed Downlink Packet Access (HSDPA), an enhanced data transmission scheme of WCDMA, is designed to increase the downlink peak rate to $8 \sim 10 \mathrm{Mbps}$ for best effort data packet transmissions [1]. In HSDPA, each physical downlink channel is comprised of a data channel and a control channel. The data channel can support several logical data channels (i.e., calls) for different users. Data from different users are then multiplexed over the physical channel and transmitted in the downlink direction.

In CDMA systems, the utilization of a channel is enhanced via assigning codes to different senders. The number of different codes allowed on a channel is called the spreading factor (SF). Previous studies on code assignment for CDMA systems allow codes of different SF to be assigned to mobile users (e.g.. [2]). As a result, code blocking may occur. Code blocking here refers to the phenomenon when an incoming call finds no suitable code with appropriate bandwidth left for it to use, although there is sufficient total residual bandwidth. To avoid code blocking, code reallocation is performed, such that occupied codes are reassigned so as to reduce the fragmentation on the code tree.

In HSDPA, the spreading factor (SF) is fixed at 16 , which means code allocation can only be done at the same SF level, i.e., $\mathrm{SF}=16$, and at most 16 codes can be allocated simultaneously. To further enhance the utilization of a channel, the Time-Domain Duplex (TDD) mode is used, in which the time domain is divided into fixed duration time slots and 16 codes are assigned to different users on a time-slot basis. Each portion allocated to each code per slot in a frame is called a mini-slot. The logical channel of a user occupies an integer number of mini-slots per frame, the allocation of which may be done continuously in the time domain, within the same time slot, or even scattered in a frame.

Code assignment is essential in the CDMA-TDD system. Each mini-slot (i.e., code) is assigned to a user and the user of each minislot should be informed of the assignment in each time slot via the control channel before the beginning of data transmission on the data channel. In HSDPA, the maximum number of bits reserved for code assignment transmitted on the control channel in each time slot is limited to 64 bits [1]. Without careful design of code assignment, this bit-space limitation may cause the HSDPA system to function inefficiently when the number of mobile nodes becomes large. This is because the SF of HSDPA is fixed at 16 , giving $N^{16}$ possible combinations to assign 16 codes to $N$ mobile users in a time slot (i.e., $N$ possible assignment per code). As the number of mobile nodes becomes large, the number of possible combinations increases rapidly, causing the requirement of more bits for code assignment on the control channel to accommodate all the combinations. Thus, from the perspective of low control overhead, the number of mobile users should be maintained small. On the other hand, when the number of mobile nodes is small, the utilization of the data channel is low, wasting the system resource. Therefore, from the perspective of high utilization, the number of mobile users should be large. These conflicting considerations motivate our work.

In this paper, a segment-based code assignment mechanism is proposed to balance the conflict between the utilization of the data channel and the overhead of the control channel for CDMA-TDD HSDPA systems. There is much work on code assignments in CDMA code trees, such as [2-4]. However, all existing code assignment mechanisms are inadequate for HSDPA systems, because they all assume that the spreading factors of the radio interface are changeable (i.e., not a fixed

This work was supported in part by the MOE program for Promoting Academic Excellence of Universities under grant number 89E-FA06-2-4-7, and in part by the National Science Council, Taiwan, under grant number NSC 91-2213-E-002-048. 
value as in HSDPA). In addition, they do not consider the TDD mode in their assignment. This calls for a new code assignment mechanism for CDMA-TDD systems with fixed SF such as. HSDPA. In our proposed mechanism, codes at the SF of 16 are grouped into a set of segments. Since the number of codes in each segment is reduced, the corresponding signaling overhead is also reduced. The load of each channel is then balanced in each segment so as to maximize the system utilization. Therefore, we can significantly increase the system performance while reducing the control overhead.

The rest of the paper is organized as follows. Section II describes the proposed code assignment mechanism. Section III shows the simulation results to demonstrate the strength of our mechanism. Finally, the paper is concluded in Section IV.

\section{SEGMENT-BASED CODE ASSIGNMENT}

To reduce the overhead of code signaling, it is necessary to reduce the possible combinations of code allocation to be accommodated by the control channel. Our solution to this problem is to group a number of codes into a segment. Since the number of codes in a segment is smaller than 16, the possible number of (code, node) combinations is also reduced. This grouping however will lower the system utilization. To enhance the utilization, the load of each segment should be balanced. In the following, we will demonstrate how our mechanism provides balanced segments for CDMA-TDD systems.

\section{A. Unit-channel and Segment}

The basic unit of allocation to each call (i.e., logical channel) in our mechanism is defined as a unit-channel, which may be comprised of multiple mini-slots per frame. Each logical channel is then allocated different number of unitchannels to meet the different QoS requirements of calls.

In our mechanism, an integer number of codes are grouped into a segment. Assume that each segment has a group of $S$ codes. Thus, there are totally $16 / S$ segments in the system. Recall that $N_{\max }$ denotes the maximum number of unitchanneis supported in the system. The maximum number of unit-channels organized in a segment, denoted as $N_{s}$, can then be expressed as $N_{s}=N_{\max } \times \frac{S}{16}$. The load of a logical channel is measured in the number of unit-channels and is distributed to any segment in which the total number of allocated unitchannels is less than $N_{s}$. Each allocated unit-channel can only be assigned codes from the code set associated with the segment to which it belongs. For example, a unit-channel in segment $i$ with a code set $C_{i}=\{0000,0001,0010,0011\}$ can only be assigned codes from the code set $C_{i}$.

\section{B. The Size of a Segment}

The value of $S$ can be determined as follows. Assume that the channel error rate of each unit-channel is mutually independent. Let $p$ denote the error rate of each unit-channel in a time slot, and let $U$ be the target utilization we would like to achieve. Thus, $1-p^{N_{s}}>U$, where $N_{s}$ is the number of unitchannels in a segment. This leads to $N_{s}>\log _{p}(1-U)$. Since $N_{s}=N_{\max } \times \frac{S}{16}$, we can derive $S>\frac{16 \log _{p}(1-U)}{N_{\max }}$, i.e., the lower bound of $S$.

The upper bound of $S$ can also be obtained, provided that the maximum number of bits reserved for code assignment on the control channel is given. In each segment, the possible combination per code is $N_{\max } \times \frac{S}{16}$. The total combination of signaling in the system is then $\left(N_{\max } \times \frac{s}{16}\right)^{16}$. Thus, the number of bits required for total signaling is $\log _{2}\left(N_{\max } \times \frac{S}{16}\right)^{16}=16 \times\left(\log _{2} N_{\max }+\log _{2} S-4\right)$. Let $n$ denote the maximum number of code bits. Thus, $16 \times\left(\log _{2} N_{\max }+\log _{2} S-4\right)<n$, i.e., $S<\frac{16 \times 2^{\frac{n}{16}}}{N_{\max }}$.

\section{The Trade-Off between Control Overhead and Svstem Utilization}

In our mechanism, $N_{s}$ is defined as the maximum number of unit-channels belonging to a segment, and $N_{s}=N_{\max } \times \frac{s}{16}$. This parameter $N_{s}$ is closely related to both the utilization of the data channel and the overhead of the control channel. As we have already shown that the system overhead is $\left(N_{\max } \times \frac{S}{16}\right)^{16}$, i.e., $N_{s}{ }^{16}$. Thus, the systems with the same $N_{s}$ will incur the same control overhead. The systems with the same $N_{s}$ also have the same maximum utilization as $1-p^{N_{s}}$.

\section{Code Assignment to Balance Segments}

In practice, mobile users may arrive and depart a cell dynamically. As a result, the number of allocated unit-channels in a segment may change from time to time, which may degrade the overall system utilization due to an unbalanced system. To improve the performance, one may consider balancing the load of each segment. The detail operation is described as follows.

The base station keeps track of the number of allocated unit-channels in each segment. 
(1) When a call arrives, the code assignment process begins.

(i) The system first determines the number of unitchannels the call requires. Assume that the call requires $k$ unit-channels.

(ii) The base station identifies the segment with the least number of allocated unit-channels, and then assigns one unit-channel in the segment to the call.

(iii) Repeat step (ii) until the $k$ unit-channels requested the arrival call all have been assigned to segments.

(2) When a call is finished, the code reallocation process for segment balancing begins.

(i) The base station identifies the segments with the largest and smallest numbers of allocated unit-channels, say segments $A$ and $\mathrm{B}$. If $\left(N_{A}-N_{B}\right)>1$, a unit-channel is moved from segment $A$ to segment $B$.

(iii) Repeat steps (i) and (ii) until for any segment in the system, say $P$ and $Q$.

\section{PERFORMANCE EVALUATION}

In this section. we describe the simulation conducted to evaluate the performance of our mechanism. In our simulation, we focus on a single base station with a fixed number of unitchannels. The two-state Markov channel model is used to model the fading condition of each channel. Let $p_{s}$ be the probability that the next time slot is in the good state given that the current time slot is error-prone, and let $p_{c}$ be the probability that the next time slot is error-prone given that the current slot is in the good state. State $G$ means the channel is good, and state $B$ means the channel is error-prone. Then the steady state probabilities $P_{G}$ and $P_{E}$ in the good and errorprone states, respectively, are given by

$$
P_{G}=\frac{p_{g}}{p_{g}+p_{e}} \text { and } P_{E}=\frac{p_{e}}{p_{g}+p_{c}} .
$$

We first observe the impact of segment size on the system utilization. In this experiment, the values 'of $p_{g}$ and $p_{e}$ are randomly selected in the range $[0.1,0.9]$. The scheduling discipline in each segment is based on ETF. We fix the total number of codes at 16 , and vary the configuration of the system from $16^{*} 1$ to $1 * 16$. In Fig $1, A^{*} B$ on the $\mathrm{x}$-axis represents a system with $A$ segments with $B$ codes each segment. There are two sets of curves in the figure. The dashed curves are collected from simulation and the solid curves are from the analytical result derived in Sec. II-B. The two curves of each set represent two different $N_{\max }$. The one with squares is based on a system with 16 unit-channels and the other (i.e., that with diamonds) is based on that with 32 unit-channels. The utilization is defined as the probability of a successful transmission per time slot.
We find that the utilization of the data channel increases as the size of a segment $S$ increases, thanks to more chances to be served or compensated in a later slot when some unit-channels are error-prone during transmission. However, when $S$ exceeds a certain value ( 8 in this example), the utilization curve becomes flat but control overhead grows dramatically as shown in Fig. 2. The value of $S$ is set to $1,2,4,8$. and 16 in this simulation. Thus, $N_{s}$ of the systen with 16 unit-channels corresponds to 1,2,4,8, 16; while $N_{s}$ of another system with 32 unit-channels corresponds to 2. 4. 8, 16, 32. From the result we can see the utilization of $8 * 2$ in the 16-channel system is equal to $16^{*} 1$ in the 32-channel system. This means that the same $N_{s}$ has the same utilization.

Fig. 2 shows the relationship between the control overhead and system utilization via simulation. We find that the curves of the utilization vs. control overhead for both the 16 and 32 channel systems are very similar, verifying our argument that the systems with the same $N_{s}$ (and with the same error condition $p$ ) will have the same overhead and utilization.

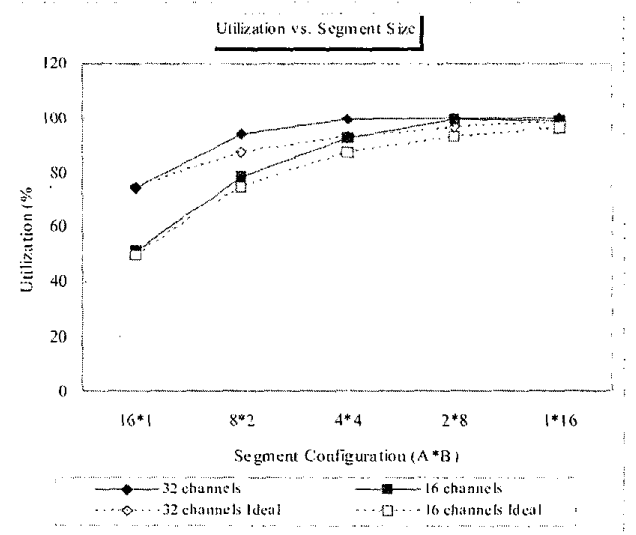

Figure 1. Segment size vs. utilization.

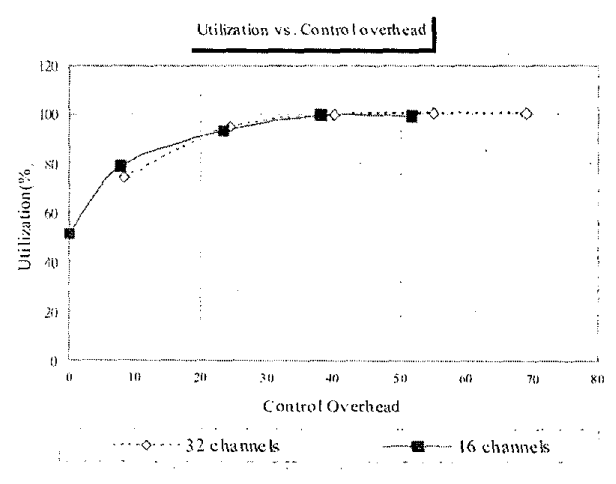


The $14^{\text {th }}$ IEEE 2003 International Symposium on Personal, Indoor and Mobile Radio Communication Proceedings

Figure 2. Uțilization vs. control overhead

\section{CONCLUSION}

In this paper, we have proposed a segment-based code allocation mechanism for CDMA-TDD HSDPA systems. We start with the description of the tradeoff between system utilization and control overhead in HSDPA, and suggest a solution. We calculate the upper and lower bounds of the size of a segment to satisfy the target utilization, and propose an approach to balancing the load of channels in each segment. Our mechanism can work with any schedulers designed for HSDPA such as [5] to lower their control overhead.

\section{REFERENCES}

[1] 3GPP TR 25.855 V2.0.0, Sep. 2001

[2] T. Minn and K.-Y. Siu, "Dynamic Assignment of OVSF Codes in WCDMA," IEEE Journal on Selected Areas in Communications, vol. 18 no. 8 , Aug 2000.

[3] C. E. Fossa, Jr. and N. J. Davis IV, "A Dynamic Code Assignment Algorithm for Quality of Service in 3G Wireless Networks," Proc. IEEE WCNC 2002.

[4] R.-G. Cheng and P. Lin, "OVSF Code Channel Assignment for IMT2000," Proc. IEEE VTC 2000.

[5] Y. Cao and V. O. K. Li, "Scheduling Algorithms in Broad-Band Wireless Networks," the Proc. of the IEEE, vol.89. no.1. Jan 2001. 